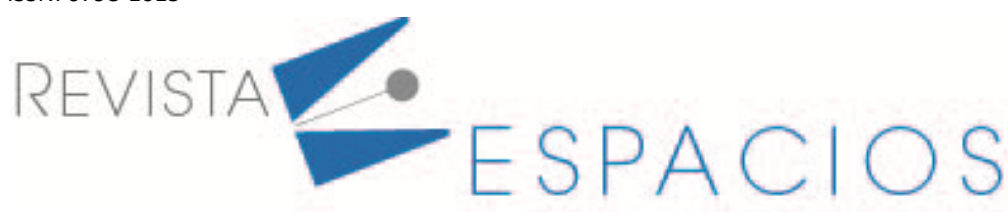

Vol. 41 (49) $2020 \cdot$ Art. 3

Recibido/Received: 09/08/2020 • Aprobado/Approved: 19/09/2020 • Publicado/Published: 23/12/2020

\title{
Una propuesta de modelo para favorecer la construcción de significado robusto de volatilidad
}

\section{A model to favor the construction of a robust meaning of volatility}

\author{
DIAZ, Miguel, A. ${ }^{1}$ \\ LUDEÑA, Carenne, C. ${ }^{2}$ \\ PEREZ, Luis, F. ${ }^{3}$
}

\begin{abstract}
Resumen
En este artículo presentamos la propuesta de un modelo didáctico diseñado para favorecer la construcción del significado robusto de volatilidad en los estudiantes de carreras administrativas, económicas y contables, mediante estrategias innovadoras como la gamificación y la solución de problemas retadores por medio de la simulación. Este modelo, es el resultado de la aplicación de procesos para validar la eficacia de las estrategias propuestas con estudiantes de la Universidad Antonio Nariño, Bogotá, Colombia, en el marco de la asignatura estadística II.

Palabras clave: modelo didáctico, simulación, solución de problemas, volatilidad.
\end{abstract}

\begin{abstract}
In this paper we present the proposal of a didactic model designed to favor the construction of the robust meaning of volatility in students of administrative, economic and accounting careers, through innovative strategies such as gamification and the solution of challenging problems through simulation. This model is the result of the application of evaluative processes with students from the Universidad Antonio Nariño, Bogotá, Colombia, in the framework of the statistical subject II.

key words: didactic model, simulation, troubleshooting, volatility.
\end{abstract}

\section{Introducción}

Uno de los motivos fuertes de este trabajo fue mostrar de forma práctica la estadística frente a la toma de decisiones importantes en particular en el marco económico, diseñando a la par estrategias pedagógicas que permitan, basados en aplicaciones financieras de relevancia y mediante el uso de técnicas de enseñanza asistidas por el computador, la comprensión de conceptos estadísticos y matemáticos abstractos tal como la volatilidad y las métricas que permiten su evaluación. De esta manera, el proyecto que da origen a este artículo, se circunscribe a carreras administrativas económicas y contables porque es a ellos los que atañe el tema de la volatilidad aplicada a las finanzas; más específicamente en estudiantes de la Universidad Antonio Nariño, Bogotá, Colombia, en dos escenarios distintos: uno es el curso de estadística II diurno en la sede Nicolás de Federman y otro es en el curso de estadística II Nocturno en la sede Sur. Se escoge el tipo de muestreo por conveniencia, como se indica en Casal (2003).

\footnotetext{
${ }^{1}$ Docente de planta. Departamento de matemáticas. Universidad Antonio Nariño. Colombia. migueld@uan.edu.co

2 Docente investigadora. Departamento de matemáticas. Universidad Central de Venezuela .Venezuela. carin.ludena@gmail.com

${ }^{3}$ Docente de planta. Departamento de matemáticas. Universidad Antonio Nariño. Colombia. luisfperez@uan.edu.co
} 
La volatilidad como concepto está íntimamente asociado a la valoración de activos, ya que se toma como un indicador que mide la variabilidad en las trayectorias temporales de cualquier actor en el mercado financiero así como de la economía en general; por ello su comprensión es imprescindible en la formación de los estudiantes de carreras de las ciencias administrativas económicas y contables, quienes en su ejercicio profesional tendrán que conocer las fluctuaciones del mercado y por ende su varianza a través del tiempo, con el fin de tomar decisiones basadas en modelos de riesgo.

Las simulaciones elaboradas en Excel, enmarcadas en metodologías de solución de problemas de tipo riesgoso, se llevaron al aula de estadística de una manera amable, sencilla y sin perder el rigor de la interpretación correcta de los datos. Este modelo se basó en distintas teorías en educación matemática que se constituyen en los ejes centrales de la investigación, como la resolución de problemas implementando el modelo de Bransford (1987), la educación matemática realista de Freudenthal (1986), y la teoría del aprendizaje significativo de Ausubel (1976). Estas tres teorías se fusionaron mediante el uso de simulaciones asistidas por computadora y la gamificación en el aula, que fueron un complemento ideal para que el alumno correlacionara todos estos conceptos. Estos referentes fueron capturados en el modelo pedagógico que se presenta más adelante.

\subsection{Marco de referencia.}

Los referentes teóricos fueron los pilares en los que descansa toda la actividad docente implicada en la construcción del modelo y de la propuesta didáctica, parte importante de ellos se muestran a continuación.

\subsubsection{Solución de problemas}

La primera referencia y más importante fue la aplicación de la resolución de problemas en el aula de estadística, uno de los principales objetivos es que mediante la aplicación del modelo los alumnos logren cierto grado de autonomía en la interpretación de los datos, proponiendo soluciones a problemas no rutinarios; los planteamientos de Bransford (1987), en su método IDEAL para la solución de problemas, fue el utilizado. Esto es muy necesario ya que inclusive los estudiantes con habilidades superiores en la solución de problemas utilizan un proceso estructurado, una estrategia definida o heurísticas que guían su acción y que les ayudan a visualizar con el fin de encaminarse en alguna solución; además que les permita evadir los inconvenientes y caminos que no conducen a soluciones efectivas al problema, como se muestra en Nieto (2005) y en Yunita (2019). En el aula de clase se pudo evidenciar que una vez que se muestra el problema al estudiante, emergen los significados implícitos en él; por ello el modelo en su filosofía aplica el cuasiempirismo lakatiano; sin embargo, es necesario medir de cierta forma los avances de los aprendices, identificando cada etapa del proceso y por ello se aplica el modelo IDEAL.

\subsubsection{Matemática realista y gamificación}

En segundo lugar, se tiene la matemática realista propuesta por Freudenthal (1991); para él las situaciones contextuales se definen como un "...dominio de la realidad que, en un proceso de aprendizaje dado, se revela al alumno para ser matematizado", justamente esta es la forma en que logramos aplicar esta teoría para nuestro modelo, a través de un juego muy realista llamado "Market Watch". Este Juego es un simulador de la Bolsa de Nueva York y es un excelente escenario para que los estudiantes relacionen todo el conocimiento estadístico en un entorno real; se bajaron las bases de datos para que el estudiante las "matematizara" como lo sugiere la matemática realista. Este juego se simula a través de una plataforma asistida en donde el participante tiene una cierta cantidad de dinero y tiene la posibilidad de invertir en acciones como si realmente estuvieran en el mercado de valores. Desde el inicio de juego, el estudiante queda inmerso en un ambiente muy competitivo entre sus compañeros ya que las consecuencias de sus decisiones se reflejan al día siguiente. El objetivo es lograr la mejor rentabilidad frente a los demás participantes. 
Un acercamiento a la definición adaptada de distintos artículos acerca de gamificación en el aula es: "La incorporación de elementos del juego en contextos que no lo son", esta es una adaptación principalmente de Huang (2013) y Such (2018). En Markopoulus (2015), se afirma que una vez puesta en marcha la gamificación, salen a la luz algunas características medibles como compromiso, influencia, lealtad, etcétera, estas caracterizaciones las convertimos en elementos de motivación.

\subsubsection{Aprendizaje significativo y cuasiempirismo}

Posterior a ello pero no menos importante, es la aplicación del aprendizaje significativo, debido a que en cada etapa del proceso se tiene en cuenta los conocimientos previos del estudiante, y aunque se pretende enseñar en base a problemas no rutinarios, se debe entregar un conocimiento, de la manera que Ausubel (1983) denomina "Aprendizaje por recepción" en la medida que algunos de los modelos a enseñar no son para ser conjeturados en el aula de clase como lo propone Lakatos (1986).

Justamente estos preceptos del cuasiempirismo lakatiano son parte esencial de este proyecto y por ello más adelante se relaciona como orientación filosófica del modelo, pero por la densidad de los temas a impartir es imposible dar el tiempo suficiente para que algunos conceptos sean conjeturados, es muy necesario aplicar las teorías de Ausubel para llegar al estudiante con temas impartidos por recepción. Basta con citar: "El aprendizaje por recepción, si bien es fenomenológicamente más sencillo que el aprendizaje por descubrimiento surge paradójicamente ya muy avanzado el desarrollo y especialmente en sus formas verbales más puras logradas, implica un nivel mayor de madurez cognoscitiva." (Ausubel,1983, p.1). El aprendizaje con enfoque constructivista del significado como se concibe en el cuasiempirismo, particularmente para algunos de los conceptos a enseñar como el movimiento browniano geométrico o el modelo de Black y Scholes, en el caso nuestro está orientado a la aplicación, no a la construcción en sí de los modelos.

\subsubsection{Modelos didácticos}

Todos los temas anteriormente descritos se plasman en el modelo propuesto. Este grupo de investigación se basa en el criterio de que los modelos didácticos están estrechamente relacionados con el proceso de enseñanzaaprendizaje. Flórez, afirma que los modelos pedagógicos modernos responden particularmente a cinco interrogantes, “... a) a qué tipo de hombre se desea formar, b) Cómo o con qué estrategias metodológicas; c) a través de qué contenidos, entrenamientos o experiencias; d) a qué ritmo debe adelantarse el proceso de formación; y e) quién predomina o dirige el proceso, si el maestro o el alumno" (Flórez, 1994, p.165); con base en ello, se diseña el modelo que responde los interrogantes que propone Flórez; además, se diseñó una metodología que evaluó la efectividad del aprendizaje como se muestra en el acápite de resultados.

\section{Metodología y propuesta didáctica}

Una vez seleccionada la muestra, se entregaron dos cursos de la asignatura estadística II (o estadística inferencial) para carreras de ciencias administrativas económicas y contables de la universidad Antonio Nariño, en Bogotá Colombia; al docente responsable de aplicar el modelo para favorecer la construcción del significado de volatilidad, mediante la propuesta didáctica que se muestra en la figura 1.

La propuesta didáctica, fue el instrumento mediante el cual se puso en marcha el modelo, el insumo necesario para que el modelo lograra cumplir con el objetivo de favorecer la construcción del significado de volatilidad, y ésta surgió después de analizar las cuestiones teóricas y el estado del arte acerca de la educación estadística en carreras administrativas económicas y contables, específicamente en el tema de la volatilidad. Cada actividad se muestra a modo de problema reto en contextos reales para que los conceptos a enseñar emerjan espontáneamente en el aula mientras el estudiante conjetura soluciones aplicando el falsacionismo lakatiano en todo momento, como se muestra en la metodología de los programas de investigación científica y como el mismo Lakatos afirma acerca de la continuidad del desarrollo científico: "... con un centro firme, convencionalmente 
aceptado y con una heurística positiva que defina problemas, esboce la construcción de un cinturón de hipótesis auxiliares, prevea anomalías y las transforme en ejemplos victoriosos; todo ello según un plan preconcebido."(Lakatos,1982.p.25). Esa misma mecánica de "heurísticas positivas y negativas" fueron las que se aplicaron en las ocho actividades, mediante preguntas orientadoras, pruebas mostradas por los estudiantes, refutaciones a esas mismas pruebas, inclusive refutaciones a las pruebas del docente. Esta manera de interacción en el aula de clase se muestra maravillosamente en Lakatos (1986).

Figura 1

Propuesta didáctica

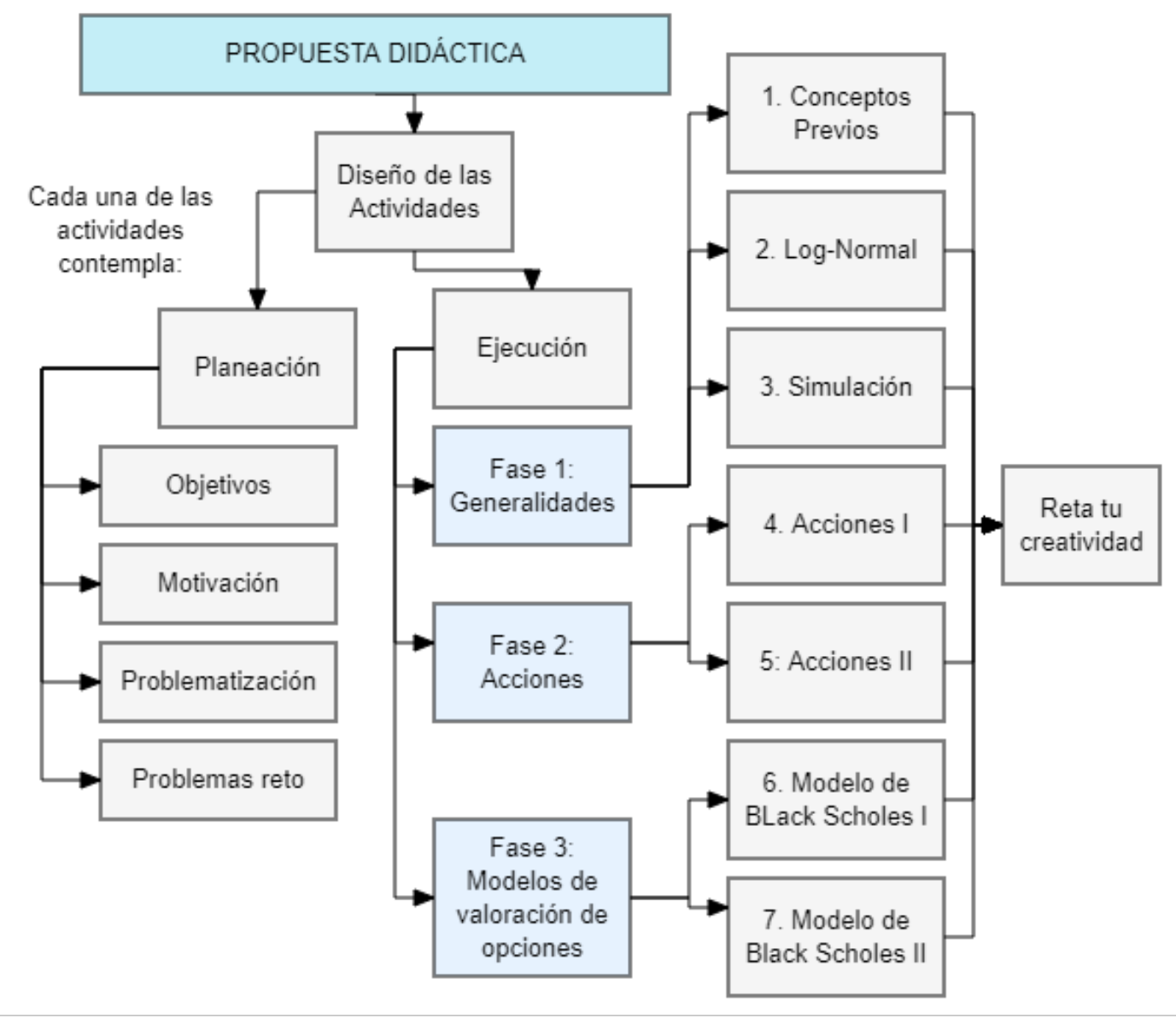

Fuente: Elaboración propia.

Es de aclarar que todos los problemas propuestos en las actividades fueron diseñados con el fin de favorecer la construcción del significado personal robusto de la volatilidad, por ello es preciso definir "robusto" como: "la construcción de redes conceptuales que desarrolla el estudiante para dar solución a problemas no rutinarios". Pérez (2015). En ese sentido, para dar solución a los problemas propuestos seguramente hubo que acudir a otras disciplinas de la matemática distintas a la estadística como la teoría de números, las series infinitas, el cálculo, la computación, etcétera.

Se aplicaron ocho actividades que se describen someramente así:

\section{Conceptos previos}

En esta actividad se elaboró un empalme con los conocimientos previos de los estudiantes, con el fin de que la experiencia en el aula le fuera significativa, como afirma Ausubel: "El factor más importante que influye en el 
aprendizaje es lo que el alumno ya sabe. Averígüese esto y enséñele consecuentemente" (Ausubel,1976, p.1); esta actividad contempló un repaso de la distribución normal, mediante problemas reto y en contexto.

\section{Log-Normal}

Aquí se desarrolló un tema de suma importancia para las ciencias administrativas económicas y contables. Llama poderosamente la atención a este grupo de investigación, el poco énfasis que se le da a esta distribución inclusive ausencia total en los currículos universitarios de estas carreras, por lo menos en el caso colombiano, ya que por lo general se toma la distribución normal para medir el comportamiento de la desviación estándar de los rendimientos financieros y ésta presenta algunas fallas, como lo afirman estudios de medición del riesgo y en (McNeil, 2015, p.85), se puede ahondar acerca del tema con suficiencia.

\section{Simulación}

En esta actividad se desarrolló la temática del movimiento browniano aritmético mediante simulaciones asistidas con Excel utilizando los pecios de las acciones que cada aprendiz ha comprado en el juego "Market Watch" con el fin de que, mediante problemas no rutinarios, ellos intuitivamente identifiquen el error al tratar esta variable con el modelo propuesto; es decir, lo utilizamos como heurística positiva siguiendo un plan preconcebido como lo propone Lakatos. Posterior a ello se les presenta el modelo geométrico, los aprendices tuvieron que variar parámetros en distintos escenarios y proponer nuevas conjeturas al problema dado.

\section{Acciones I}

En esta actividad se profundizó en el tema de la covarianza utilizando el mercado accionario, con el fin de que utilizaran los índices beta con suficiencia en el momento de escoger entre distintas acciones. La idea principal fue afianzar características inherentes a la volatilidad mediante la práctica real, la simulación y la gamificación. Esta fue una actividad poco guiada ya que ellos estuvieron muy motivados por realizarla, se atribuye esta motivación a la competitividad que generó el juego o simplemente por curiosidad y diversión; como lo afirma Markopoulus: “...también hay beneficios de la gamificación que no se pueden medir como el simple, pero no cuantificable concepto de diversión, que es probablemente la razón principal por la que se juega." (Markopoulus, 2015, p.7).

\section{Acciones 2}

En esta actividad se les presentó problemas de pronóstico para las variables rentabilidad y precio, y este tema empalma con regresiones en el contenido programático de la asignatura estadística II en la Universidad Antonio Nariño; en esta actividad se realizan simulaciones buscando el mejor ajuste y la correcta interpretación de $r^{2}$; mediante la ayuda de Excel e internet, se elaboraron problemas retadores de envergadura superior a los que se pueden presentar en el aula con el recurso limitado de la calculadora científica.

\section{Modelo de Black Scholes I}

Este modelo se presenta en su forma más simplificada, mostrando el precio de la opción como variable aleatoria; este comportamiento "extraño" del precio de los subyacentes, fue el primer paso para que el estudiante hiciera las simulaciones necesarias para identificar las conexiones entre varianza a través del tiempo en instrumentos distintos a las acciones.

\section{Modelo de Black Scholes II}

Esta actividad tuvo como objetivo, simular los escenarios posibles en los que los inversores, en óptimas condiciones de volatilidad; en ella los aprendices escogieron entre compra de activos de distintas índoles como oro, acciones, opciones, etcétera. Ya el estudiante en esta actividad viene con una concepción de la volatilidad muy madura y el significado personal construido de manera apropiada. 


\section{Reta tu creatividad}

Esta actividad fue, como su nombre lo indica, un reto para los estudiantes más brillantes, no constitutivo de calificación dentro de las actividades regulares de la asignatura; se entregó desde el inicio del curso y durante el semestre ellos hicieron aportes a la solución de problemas de un nivel muy alto, y ante los avances, se bonificó la nota parcial en el curso regular.

Esta propuesta metodológica inició con una prueba de entrada y finalizó con otra de salida, con el fin de realizar pruebas de contraste y para ello se escogió una prueba no paramétrica, que se puede visualizar en Wilcoxon (1970), y se describe en este mismo artículo en la sección de resultados.

\section{Modelo propuesto para favorecer la construcción del significado robusto de volatilidad}

Esta propuesta facilitó la construcción del significado robusto de la volatilidad en un contexto real y así favoreció el razonamiento crítico y cuantitativo de los estudiantes de ciencias administrativas, económicas y contables, y en la síntesis de todos los procesos que conllevan a la resolución de un problema en el campo de las finanzas, mediante el uso de la estadística, específicamente la volatilidad. En el modelo se definen dos fases significativas:

1. la Fase de Planeación: En ella se encuentra una serie de actividades que están al margen del aula, aquí no se involucra a los aprendices objeto de estudio, las etapas se concretan mediante la interiorización de la problemática propuesta por parte del docente y la asignatura a impartir; estas son: la orientación, la concreción y la valoración del estado inicial.

2. La Fase de aplicación o ejecución: En esta segunda fase se surte una serie de etapas experimentales, que son la puesta en marcha del proyecto con los estudiantes, aquí se debe generar una construcción robusta y paulatina del concepto de volatilidad estadística, alimentada con los constructos nombrados en las actividades y los elementos propios de las teorías que se utilizan como mediadores pedagógicos, que son la salida del modelo. Las etapas son: Integración, preevaluación, ampliación y evaluación. El modelo en su totalidad se muestra en la figura 2.

Figura 2

Modelo Propuesto para favorecer la construcción del significado robusto de volatilidad.

\section{Modelo:}

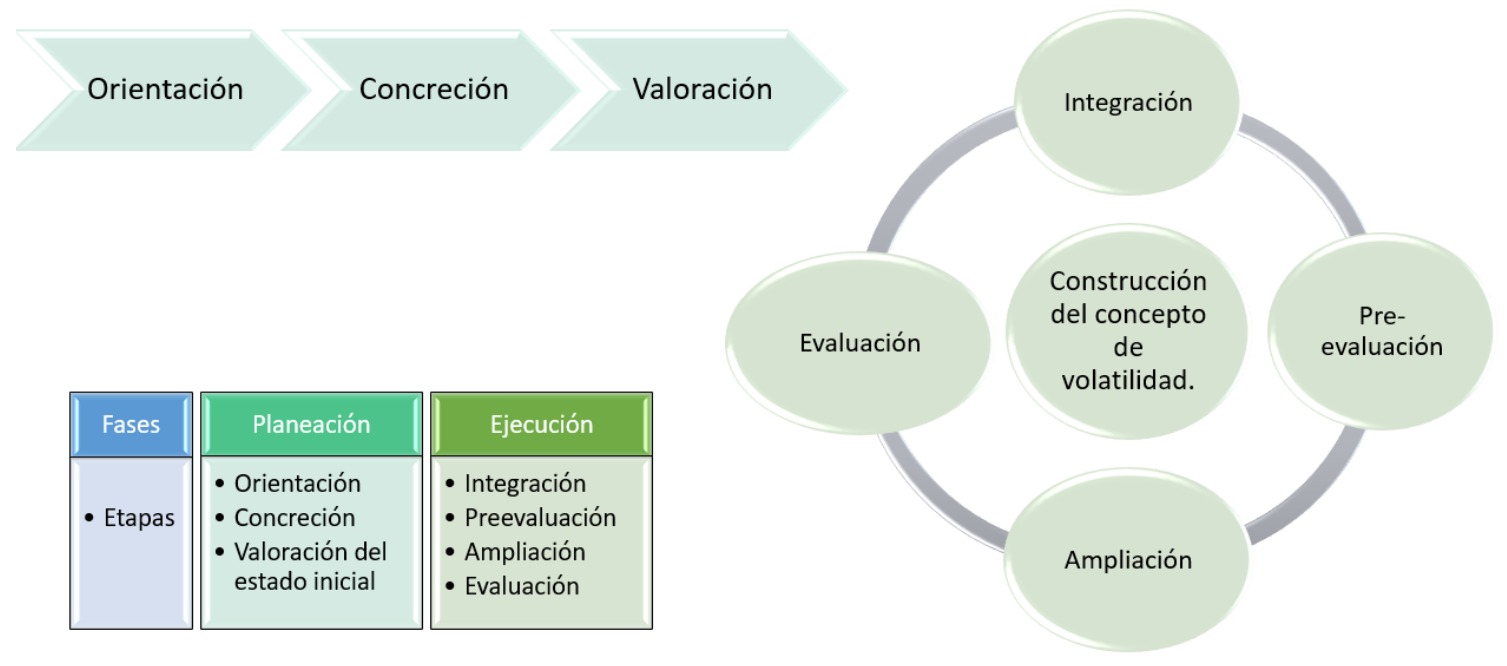

Fuente: Elaboración propia. 
Como centro de las etapas experimentales emerge el constructo del significado personal de volatilidad que se incrementa en cada actividad de forma paulatina. A continuación, se describe cada una de las etapas del modelo.

\subsection{Orientación}

La etapa de orientación tiene como fin dar el sustento teórico, filosófico y disciplinar. El docente debe garantizar que se aborde los conceptos estadísticos necesarios para aplicar las actividades que conlleven a la construcción del significado robusto de volatilidad mediante los problemas retadores y con simulación. Además de ello se deben conocer los fundamentos teóricos de la estadística, los pilares filosóficos de la propuesta y los referentes pedagógicos para dirigir el proceso de formación del significado de volatilidad. El trabajo del docente consiste en organizar en los conceptos que fomenten la construcción del significado robusto de volatilidad estadística.

Esta etapa tiene unas subetapas bien definidas que se muestran en la figura 2, y a continuación se describen así:

\section{Orientación teórica}

Para la buena utilización del modelo, es necesario que el docente tenga un horizonte teórico claro el cual debe seguir, en este caso se sugiere la teoría de solución de problemas retadores, la gamificación en la educación, el uso de la tecnología en el aula, el aprendizaje significativo y la matemática realista, para este grupo de investigación son fundamentales las teorías antes nombradas.

\section{Orientación disciplinar en estadística y finanzas}

Para los docentes que imparten la asignatura estadística II, las temáticas generalmente son las siguientes: Repaso de distribuciones discretas y continuas, muestreo, distribuciones en el muestreo, intervalos de confianza, prueba de hipótesis, regresión lineal y análisis de varianza. En cuanto a las temáticas a impartir es necesario que además de los temas antes nombrados, el docente tenga un horizonte claro ante las nuevas temáticas como son: Simulaciones, movimiento browniano, futuros financieros, acciones, opciones etcétera. De ese modo es muy importante que el docente que decida aplicar el modelo conozca los apartes de la teoría financiera a utilizar.

Figura 2

Etapa de Orientación

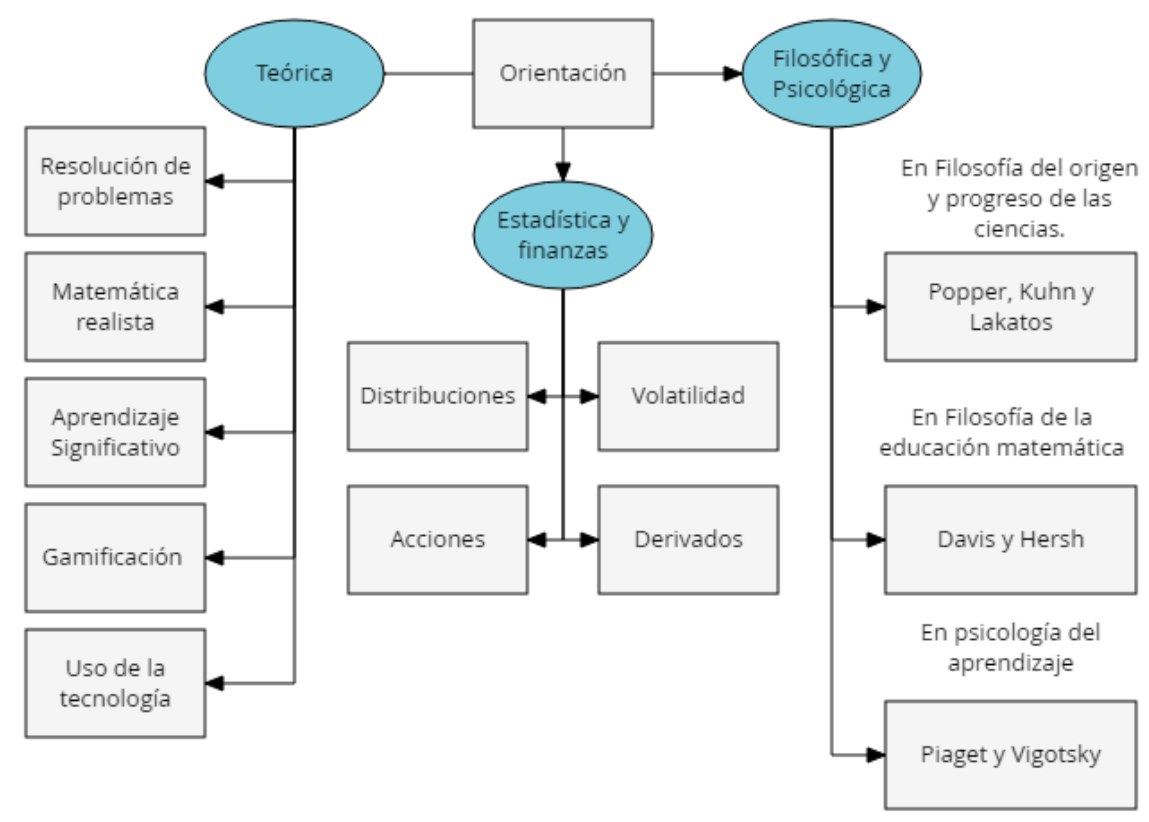

Fuente: Elaboración propia 


\section{Orientación psicológica y filosófica}

La orientación psicológica de este trabajo está basada en Piaget (1976) con la teoría del desarrollo cognitivo, más allá de las etapas cognoscitivas en las cuales Piaget dividió el desarrollo del ser humano; este trabajo tiene como horizonte el constructivismo propuesto por él y la forma de hacer matemáticas desde la intuición, a pesar de que se utiliza el ordenador y sus herramientas como Excel y Geogebra, la idea es que la propuesta gire en torno a la solución de problemas retadores en un ambiente competitivo. Otro punto de vista bien importante, lo constituye la forma en que se induce al estudiante a la zona de desarrollo próximo como lo propone Vigotsky (1988), entendiendo a esta zona como todo aquello que el estudiante sería capaz de hacer con la supervisión y ayuda del profesor, quien acompaña el proceso hasta donde sea necesario debido a la complejidad de los temas a impartir. De ese modo, es un híbrido entre las dos posturas bien interesante en donde se sigue la forma de actuar del estudiante, interviniendo únicamente en las situaciones en las que definitivamente no será capaz de llegar, por ejemplo en las etapas iniciales de la simulación; el estudiante está explorando las posibles formas de desarrollar su intuición pero su manejo del Excel no es suficiente, en éstas situaciones se debe intervenir con el fin de acelerar el proceso en cada uno de los estudiantes y nivelarlos frente a otros de sus compañeros en cuestiones triviales como el manejo de la herramienta, se concibe esta acción como llevar al estudiante a la zona próxima; una vez allí, él será capaz de resolver los problemas propuestos.

La orientación filosófica acerca del origen y avance del conocimiento científico se toma como referencia el falsacionismo del austriaco Popper (1986), desde esta postura se considera el conocimiento científico como verdadero hasta encontrar un caso donde no se cumpla, además el conocimiento es temporal hasta que se refute; por ello, se puede afirmar que un estudiante de la clase, desde el punto de vista del modelo, puede perfectamente falsear las hipótesis nulas del conocimiento que el docente pretende impartir en el aula, porque cuenta con las herramientas necesarias, (Excel, simuladores e internet); por ello, el estudiante inquieto no debe estar completamente seguro de que lo que se afirma en clase es cierto.

La refutación de una teoría, según Lakatos, depende del éxito total de una teoría rival, una nueva teoría que perfecciona la ciencia y el conocimiento, estas teorías están relacionadas entre sí y comparten un núcleo duro, con su cinturón protector constituido por hipótesis auxiliares que no permiten que el núcleo duro sea falseado. Las heurísticas, son las reglas que rigen al científico y las clasifica en dos, las positivas y las negativas. Las positivas se pueden ver como la forma y la guía de cómo hacer estadística en el aula, mediante simulación, con situaciones reales, aplicando la gamificación, etcétera. La heurística negativa justamente es lo que no se debe hacer en el aula. Por ejemplo, una clase completamente magistral con un enfoque conductista al mejor estilo de Pavlov (1927), o quizás una clase formalista del tipo Hilbertiano, como se propone en Hilbert (1928), e inclusive tratar de desarrollar en estudiantes de ciencias económicas una clase con la metodología propuesta en la escuela logicista, como se muestra en Russell (1982), y que se puede visualizar con profundidad en De Losada (2012), ya que todos estos modos de enseñanza pueden aplicar bien para el estudiante de matemáticas, pero no para los estudiantes del presente experimento.

El énfasis de la investigación es participativo mediante la resolución de problemas retadores, en ese sentido, el compromiso de los estudiantes es fundamental y progresivo ante la posibilidad de que construya su significado robusto de una forma paulatina e incremental, y que éste sea completamente significativo. Además de ello, se genera una competencia sana, cuyo fin es el de verificar cuál de las soluciones propuestas tiene menor número de fallas. Claro, toda esta metodología tiene su núcleo central que no tiene refutaciones y es el contenido estadístico

En cuanto a la aplicación de esta filosofía de clase, en el modelo propuesto se establece una etapa de preevaluación que, mediante preguntas orientadoras, llevan al estudiante a generalizaciones simplificadas en las que progresivamente aporta ideas a la solución de un problema utilizando el cuasiempirismo de Lakatos (1986); 
aquí el educando contribuye con pruebas que defiende, individual o grupalmente además de refutar las pruebas que aportan los demás; de ese modo, desde los fundamentos filosóficos del modelo, se permite que el estudiante ataque las hipótesis del docente mediante falsaciones ya que estas serán parte sustancial de la construcción del significado robusto de volatilidad, que es lo que se pretende que emerja en el aula.

Finalmente, respecto a la filosofía propia de las matemáticas, algunas ideas de Reuben Hersh serán de gran utilidad para orientar el proyecto de investigación. Él clasifica a lo largo de la historia de las matemáticas en dos corrientes: La corriente principal, que considera la matemática como sobrehumana, abstracta, ideal, infalible y eterna. La otra corriente los llama los Humanistas y rebeldes que afirman que la matemática es una creación humana, Hersh (1997); consideramos que lo que propone el modelo es abiertamente de la corriente de los humanistas y rebeldes, por todo lo descrito acerca de la mecánica de la clase y la falibilidad del profesor. Desde su gestación, este proyecto pretende que la participación del estudiante sea activa y no existe mejor manera de construir algún conocimiento propio que desde la misma base de su error.

El profesor debe generar las condiciones necesarias para que el estudiante aprenda de modo vivencial, con ensayo y error que claramente favorece la construcción del significado robusto de volatilidad desde el punto de vista crítico del aprendiz, como afirma Hersh: "Un análisis realista de la intuición matemática debe ser un objetivo central de la filosofía de la matemática" (Hersh, 1997 p.62). Esta frase la acota Hersh hablando precisamente de intuición, prueba y falibilidad; y recopila bastante de la idea principal de la orientación que pretende llevar este trabajo, la construcción del significado robusto de volatilidad basados en la intuición del estudiante, la falibilidad del profesor y la consistencia de las pruebas que aporta.

\subsection{Concreción}

Esta segunda etapa del modelo se adapta de Almira (2015) y en ella, el docente debe proponer los problemas que encaminen al estudiante hacia cada uno de los conceptos necesarios que permitan el desarrollo de la construcción del significado de volatilidad dentro de un contexto real, en un plan preconcebido que permita un aprendizaje significativo. Esta etapa también está encaminada a determinar la contradicción interna y la externa, es decir, los obstáculos y errores comunes encontrados en el aprendizaje de los estudiantes y los obstáculos evidenciados en la enseñanza respectivamente, una vez evidenciados tales obstáculos, se diseñan los problemas procurando evadirlos. En esta etapa se concreta la propuesta didáctica que será llevada al aula.

Para determinar algunos elementos visibles de la contradicción interna, fue necesario encontrar algunos factores que afectan negativamente en el proceso de aprendizaje de la volatilidad estadística y para ello, se realizaron pruebas de entrada a los estudiantes y encuestas de percepción hacia la estadística y el tema específico de la volatilidad.

Los elementos importantes de la contradicción externa, es decir, los factores que inciden negativamente en el proceso de enseñanza se recopilaron mediante encuestas con docentes expertos en la asignatura. En la figura 3 se detalla la estructura de la etapa 2. 
Figura 3

Etapa de Concreción

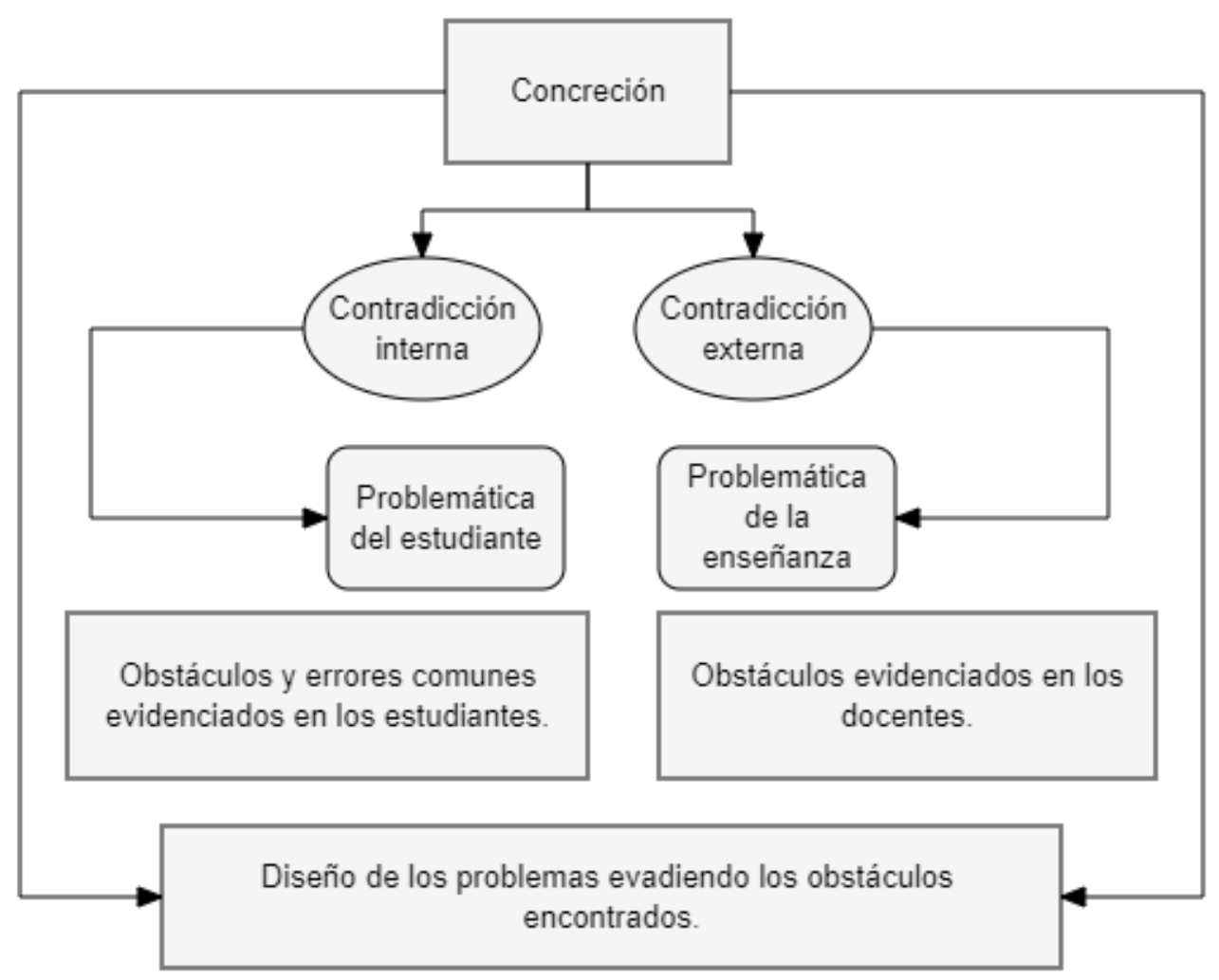

Fuente: Elaboración propia

\subsection{Valoración del estado inicial}

En esta etapa, se pretende conocer el estado actual del proceso de enseñanza aprendizaje de la estadística, en particular el concepto de volatilidad y sus aplicaciones con los aprendices objeto de estudio, y de esa forma el docente encargado de aplicar el modelo tendrá una idea de hacia dónde encaminar su enseñanza. El dominio de los conocimientos previos posibilita insertar y dirigir de manera efectiva la actividad docente, para favorecer la construcción del significado robusto de volatilidad. También es propicio para tener un punto de partida y determinar cómo influye afectiva y cognitivamente a los estudiantes los contenidos de la estadística en su desempeño en cada actividad desarrolladas en la etapa de concreción. La relación explicada se representa en el esquema de la figura 4. 
Figura 4

Valoración del estado inicial.

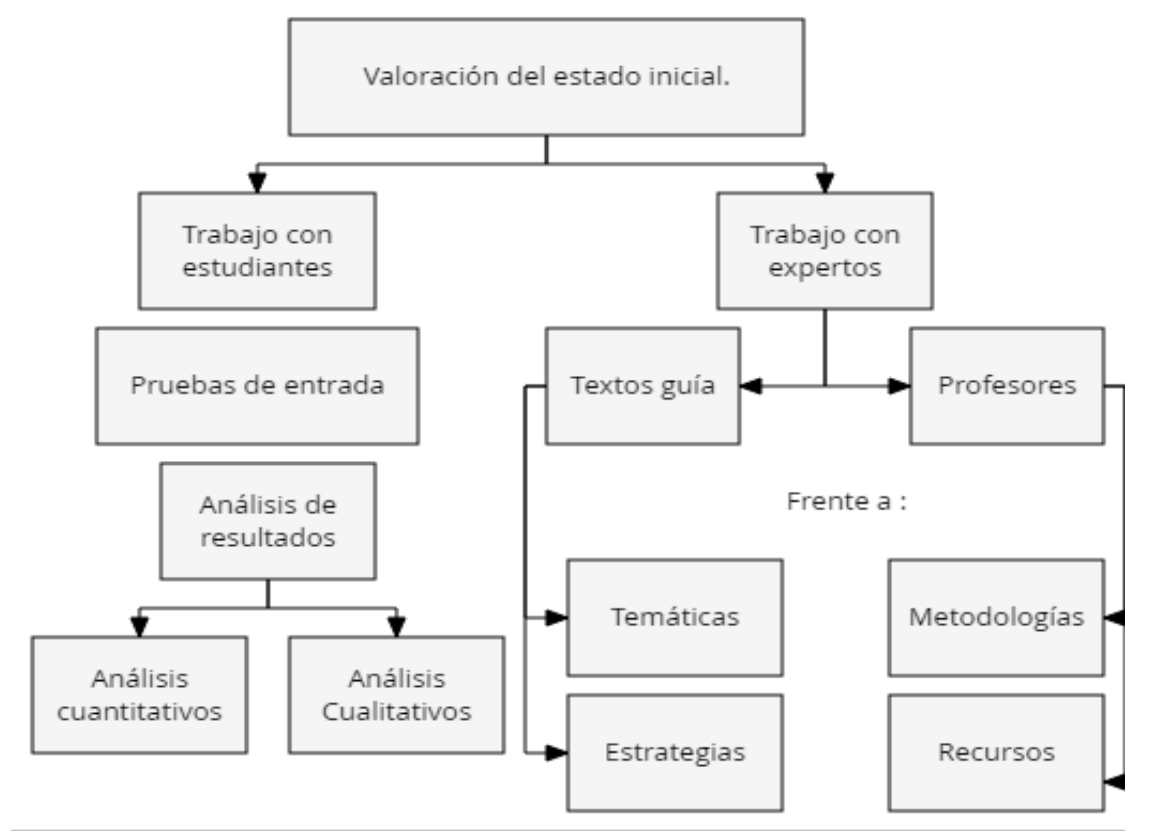

Fuente: Elaboración propia

\subsection{Integración}

Es la primera etapa de la fase de ejecución, es donde se trabaja con mayor profundidad en los elementos esenciales de los problemas retadores, la simulación y las teorías en educación propuestas. Aquí aparece el objetivo, que en gran parte depende del diagnóstico inicial efectuado en la etapa tres. El docente debe dar a conocer y hacer partícipe al estudiante del proceso, permitiendo que conozca lo que se espera de él y cuál debe ser el producto de la actividad que va a realizar.

En esta etapa se integran las teorías utilizadas que deben estar presentes en cada uno de los problemas formulados; la motivación explícita e implícita latente en cada una de las actividades y en la actitud del docente hacia sus estudiantes; además, se integra con los contenidos a impartir. La motivación aparece de forma explícita en la actividad y la posibilidad de que el aprendiz aporte ideas que den solución a los problemas propuestos, es la forma implícita de ella.

En la elaboración de los problemas se considera no sólo su posible contribución a la formación de los conceptos estadísticos previstos, sino que se aprecie su relación con el desarrollo del pensamiento estadístico variacional, desarrollo del pensamiento matemático, la recursividad con la hoja de cálculo y la motivación por la asignatura. La selección apropiada de los problemas retadores es fundamental, estos deben contener situaciones que admitan riqueza en distintas temáticas y que generen preguntas complementarias, tanto matemáticas y estadísticas, así como en su aplicación en las ciencias económicas; esto favorece la construcción de significado robusto.

\subsection{Preevaluación}

Mediante preguntas orientadoras en el proceso de enseñanza-aprendizaje; se prueba y se refuta en todo momento las soluciones propuestas por los estudiantes y esta dinámica ellos la realizan bidireccionalmente, una es frente a sus pares estudiantes y otra frente al profesor. De esa forma el docente se crea una idea generalizada de las dificultades y obstáculos en el proceso de aprendizaje, además identifica las fortalezas y la intuición natural de los estudiantes con el fin de establecer el siguiente problema reto. En esta etapa se controla el nivel alcanzado 
por los estudiantes en su desempeño frente a la resolución de problemas ya que éstas son un elemento esencial para el logro del objetivo final, para el caso nuestro, se evidencia tal nivel mediante los elementos presentes en cada prueba aplicando el modelo IDEAL de solución de problemas. Además, se percibe el nivel de desarrollo en la construcción personal de los significados estadísticos propuestos en la investigación. El tránsito entre la preevaluación y la etapa anterior se muestra en la figura 5.

Figura 5

Tránsito entre la preevaluación y la etapa de integración mediante la solución de problemas.

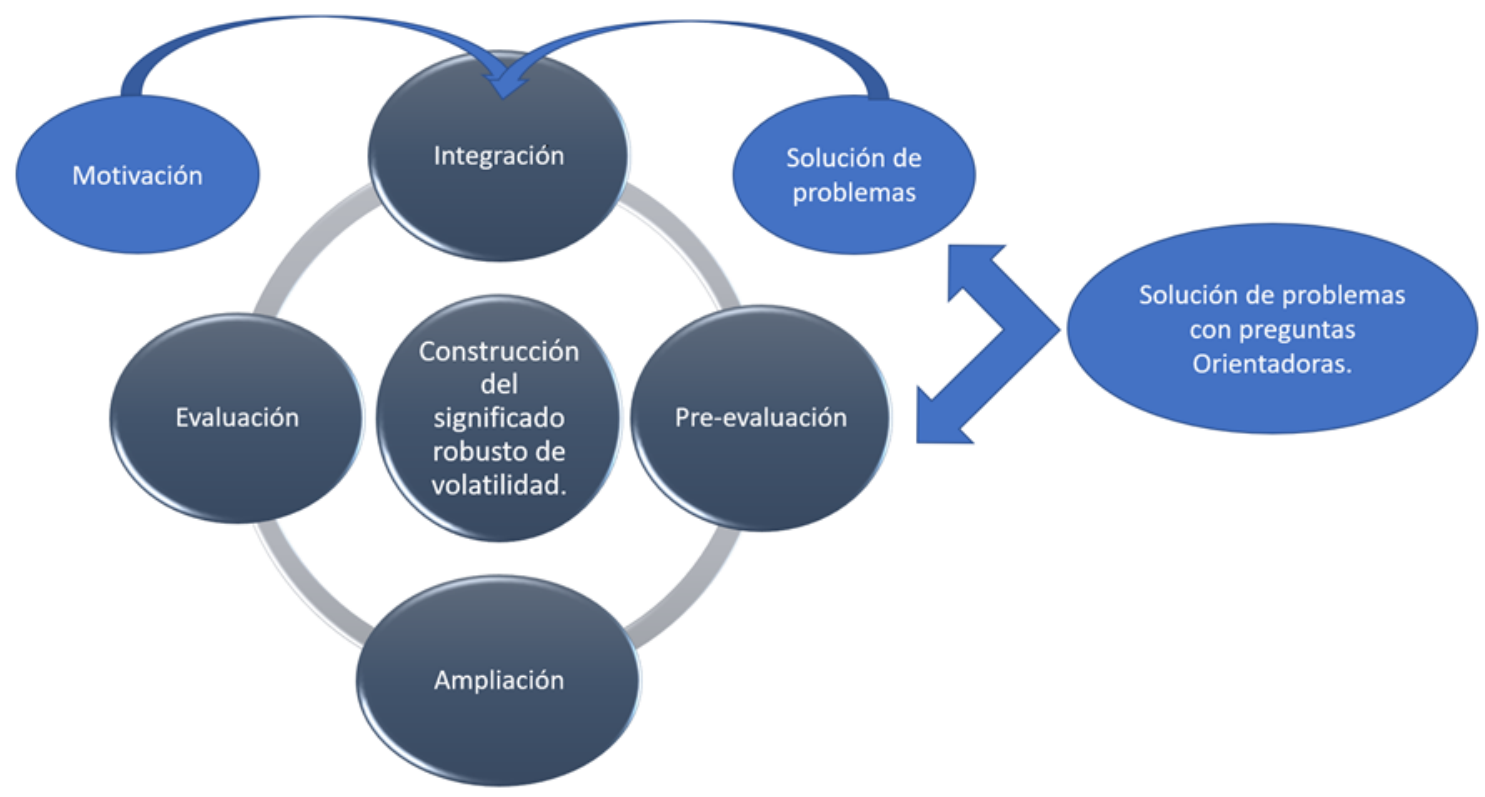

Fuente: Elaboración propia.

\subsection{Ampliación}

En la etapa de ampliación hay una correlación de los elementos que provienen de la estadística con otros elementos que le son necesarios para la solución de su problema reto, por ello el término "ampliación" hace referencia a que el estudiante debe construir más que un significado, debe construir un significado robusto de la volatilidad, en concordancia con el elemento del significado que Godino (2002), llama como Intensivo. Además de ello, la etapa número seis está orientada a la realización de selección de nuevos problemas que ataquen las deficiencias encontradas en la etapa anterior. En esta etapa es donde realmente se construye el significado robusto; docente y estudiante aprenden uno del otro y esa dinámica presentada en la preevaluación, de preguntas orientadoras en distintos sentidos; genera discusiones académicas constructivas alrededor de los temas que son necesarios para la solución de los problemas propuestos.

\subsection{Evaluación}

Esta etapa garantiza la eficiencia del modelo ya que ella se mide por medio de la entrega de los avances a la solución de las actividades. La construcción robusta del significado de cada uno de los conceptos y su aplicabilidad a la volatilidad es será la evidencia en la evaluación en cada etapa del proceso, siendo la más grande y mejor motivación para el estudiante ya que en un problema reto en donde él reconoce que ha logrado llegar a solucionar, por lo menos en parte, un problema que parece tener mucha complejidad es un motivador muy fuerte, haciendo que el ciclo se repita en cada actividad iniciando con la motivación.

El paso de la evaluación hacia la motivación mediante la solución de problemas se muestra en la Figura 6. 
Figura 6.

Tránsito entre la evaluación y la integración mediante la solución de problemas como principal fuente de motivación.

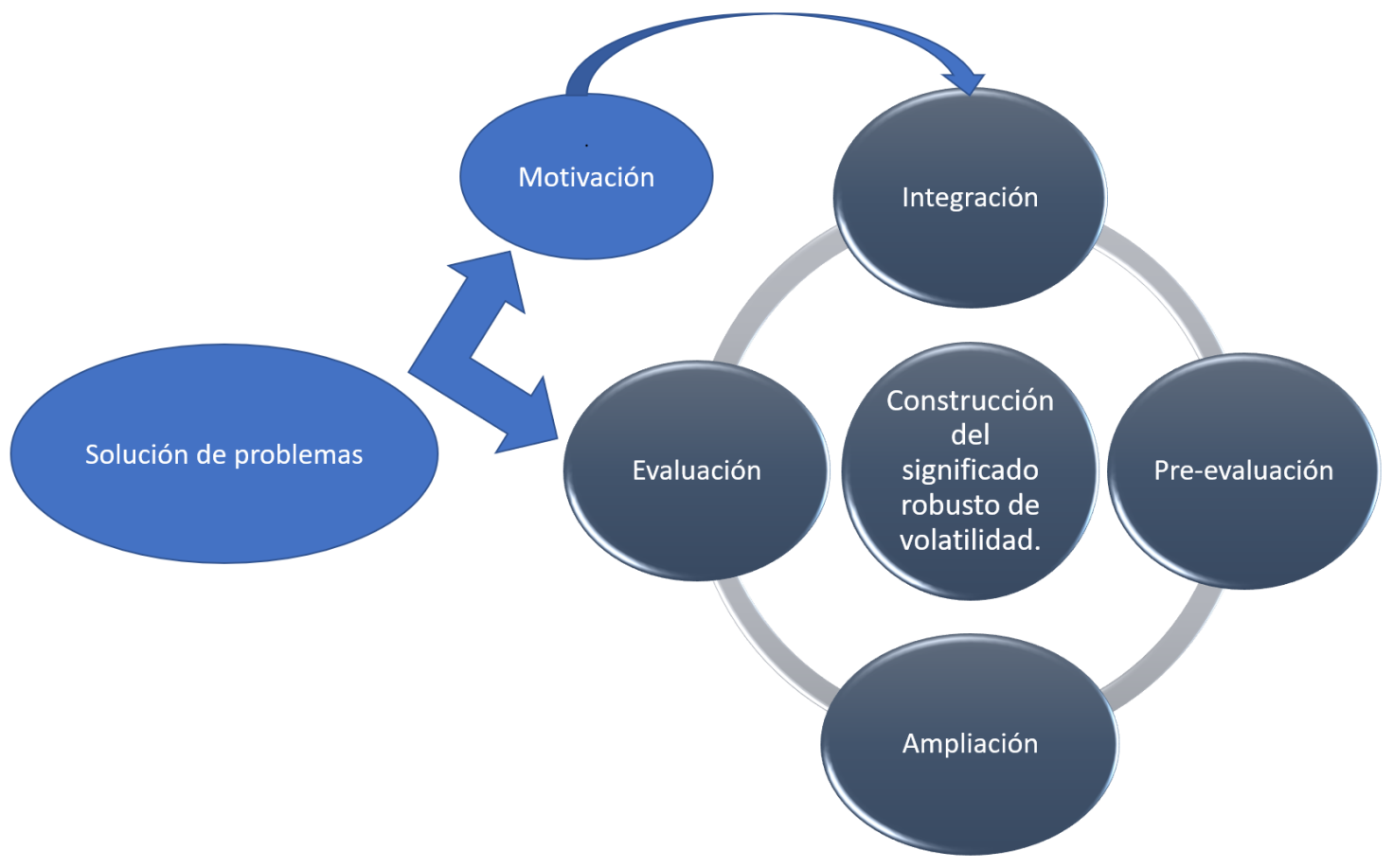

Fuente: Elaboración propia.

\section{Resultados}

Para validar el modelo se utilizaron pruebas de entrada y salida haciendo una comparación entre ellas, después de analizar y encontrar que las diferencias no son normales, y las pruebas paramétricas no modelan correctamente los resultados de los estudiantes antes y después de la aplicación del modelo, se decide utilizar la prueba de Wilcoxon (1970).

Por otro lado, se analizaron los resultados de dos pruebas acerca de las actitudes de los estudiantes respecto al estudio de la estadística y su utilidad, uno es el instrumento diseñado por Auzmendi (1992) y otro concerniente al tema de volatilidad específicamente, de diseño propio. Se decide implementar estas encuestas de percepción debido a que este instrumento incluye preguntas orientadas a la diversión por medio del aprendizaje, orientadas a la percepción de la utilidad de la estadística, a la percepción de los estudiantes referente a la seguridad que siente el estudiante al resolver un problema, y justamente esas son las bases teóricas de este proyecto. Posteriormente se aplicó la metodología utilizada para el análisis de la construcción del significado, mediante los elementos propuestos por Godino (2002), respecto a las actividades entregadas por los estudiantes. Una vez analizadas todas las pruebas se elaboró una matriz de resumen para concluir.

Finalmente, los videos obtenidos en cada una de las sesiones se analizan mediante la metodología propuesta por Planas (2006), quien diseñó un modelo de análisis de videos para el estudio de procesos de construcción de conocimiento matemático. Los resultados generales se recopilaron en una matriz de resultados para posteriormente concluir.

En la figura 7 se muestra la estructura general utilizada en la validación del modelo. 
Figura 7.

Esquema general utilizado para

la validación del modelo.

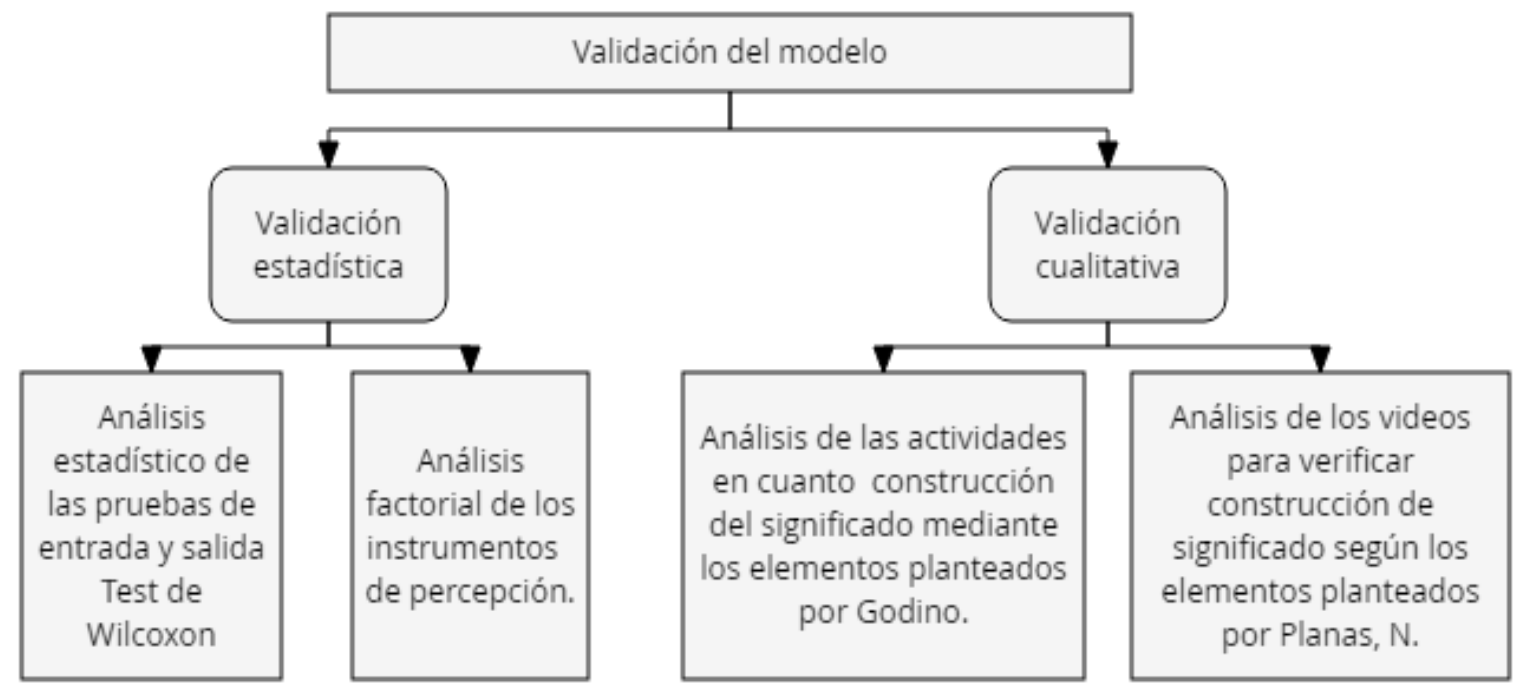

Fuente: Elaboración propia.

Los resultados a las pruebas de validación aplicadas se muestran a continuación.

1. En cuanto a la prueba de Wilcoxon, se plantean las hipótesis del caso:

$H_{0}$ : "La aplicación del modelo de enseñanza no afecta el desempeño de los estudiantes en temas relativos al tema de volatilidad"

$H_{1}$ : "La aplicación del modelo de enseñanza afecta el desempeño de los estudiantes en temas relativos a la volatilidad".

Una vez analizados los resultados según la metodología propuesta por Wilcoxon, se muestra a continuación en la tabla 1.

Tabla 1

Resultados en la prueba de los signos de Wilcoxon y contraste con el valor crítico.

\begin{tabular}{|l|c|c|c|}
\hline & Tamaño de la muestra: & $\begin{array}{c}\text { Estadígrafo de prueba: } \\
\min \left\{T_{+}, T_{-}\right\}\end{array}$ & $\begin{array}{c}\text { Valor crítico para la prueba } \\
\text { de los signos de Wilcoxon } \\
\text { con alfa de } 0.05\end{array}$ \\
\hline Sede Sur: & 22,0 & 22,5 & 65 \\
\hline $\begin{array}{l}\text { Sede Nicolás de } \\
\text { Federman: }\end{array}$ & 18 & 18 & 40 \\
\hline
\end{tabular}

Fuente: Elaboración propia.

La prueba de los signos se realizó con prueba de salida menos prueba de entrada; de esa forma se concluye que la diferencia además de ser significativa es positiva, lo que indica la mejora en la concepción del significado de volatilidad en ambas sedes. 
2. En cuanto al análisis factorial, para la prueba de Auzmendi (1992) se realiza con la metodología de análisis de componentes principales con método de rotación varimax con normalización Kaiser y se tiene que el dato más relevante es la fuerte correlación entre los factores que explican el $86,44 \%$ de la varianza, son las preguntas:

a. Me divierte el hablar con otros de Estadística.

b. Tengo confianza en mí mismo/a cuando me enfrento a un problema de Estadística

c. La Estadística es agradable y estimulante para mí

d. Me gustaría tener una ocupación en la cual tuviera que utilizar la Estadística

e. El utilizar la Estadística es una diversión para mí

Y estas preguntas son concordantes en gran medida, con las teorías utilizadas para el modelo así:

La gamificación, que explica la pregunta a y e.

El aprendizaje significativo y la matemática realista, que explica la pregunta d.

La resolución de problemas, que explica la pregunta b y c.

Es de aclarar que esta interpretación se realiza después de analizar los datos en contraste con la experiencia en el aula de clase, y seguramente presenta sesgo en su interpretación, sin embargo, de forma suficiente se infiere que la aplicación del modelo marca positivamente la percepción de los estudiantes frente a la estadística.

Por otro lado, para la prueba de percepción acerca del tema específico de volatilidad, en el diseño del instrumento se obtiene un alfa de 0,934. Esta es una prueba con 9 preguntas y en el análisis de rotación de factores Varimax, indica una fuerte correlación entre todos los factores involucrados en el instrumento, afianzando la idea que el modelo cohesiona de una manera suficiente distintos elementos como los contenidos, la motivación, la pertinencia y utilidad de la volatilidad.

3. Para el análisis de los elementos presentes en la construcción de significado propuestos por Godino (2002), se analiza en cada uno de los 29 problemas de las 8 actividades y se registra la presencia de alguna manifestación de los elementos constitutivos de construcción de significado en cada problema. El resumen de estos registros se muestra en la tabla 2.

\section{Tabla 2}

Resultado de los análisis de presencia de elementos constitutivos de construcción de significado por cada prueba

\begin{tabular}{|c|c|}
\hline Elementos de significado: & $\begin{array}{c}\text { Porcentaje de pruebas con presencia } \\
\text { del elemento constitutivo de } \\
\text { construcción del significado. }\end{array}$ \\
\hline Extensivos & $61 \%$ \\
\hline Ostensivos & $75 \%$ \\
\hline Actuativos & $79 \%$ \\
\hline Intensivos & $84 \%$ \\
\hline Validativos & $76 \%$ \\
\hline
\end{tabular}

Fuente: Elaboración propia

Indican estos resultados que la presencia de cada uno de los elementos propuestos por Godino, infiere que la construcción de significado estuvo presente en gran medida para las pruebas realizadas. 
4. Por último, cada prueba videográfica es analizada con la metodología propuesta en Planas (2006), y gratamente se evidenció, que en el $100 \%$ de los videos existen los elementos propuestos para la construcción de significados matemáticos.

Es de aclarar que las metodologías de validación se referencian, pero no se explican en su totalidad por no ser centrales en este artículo y los análisis exhaustivos de las dos pruebas anteriores, reposan en la tesis doctoral que da soporte a este artículo, en la Universidad Antonio Nariño, en la Maestría y Doctorado en Educación matemática.

\section{Conclusiones.}

La resolución de problemas es un excelente camino para empoderar a cada estudiante, el problema no debe ser un reto inalcanzable, todos podemos hacer matemáticas y bajo esa premisa, el estudiante alimentará su motivación mediante sus logros pequeños o grandes; pero propios.

La construcción de significado es el objetivo primordial, deben ser unas clases en donde los estudiantes generen preguntas y respuestas enriquecedoras, con equivocaciones y aciertos que van moldeando sus significados personales, esto forma el criterio de un buen profesional.

La gamificación en el aula motiva, vuelve al estudiante competitivo, él desea aprender más para ganar y no es una competencia insana, se hace en beneficio de su propio aprendizaje.

La matemática realista es imprescindible en los procesos de enseñanza aprendizaje, al inicio de cada curso, sea de estadística, cálculo, matemáticas básicas, etcétera; siempre surge la pregunta, ¿y eso en qué se aplica?, por esta razón, las finanzas y su abundancia de datos es una excelente excusa para hacer estadística en contexto.

La motivación del estudiante es un elemento primordial en cada proceso de aprendizaje y cada estrategia que utilicemos en el aula debe ser un camino que nos conduzca a esa motivación inclusive la misma evaluación.

La evaluación cierra el ciclo del modelo propuesto y lo inicia precisamente con la motivación, por esta razón la evaluación no debe ser un símbolo de frustración en el estudiante, debe ser un elemento de motivación ya que, el hecho de que su aprendizaje se construya en base de su mismo error inspira al aprendiz a superarse a sí mismo y no al instrumento evaluativo.

Cuando el docente se despoja de su ego de proveedor de conocimiento, cuando entiende realmente que debe aprender de sus estudiantes y que esa relación casi simbiótica es indispensable; únicamente a partir de ello, es posible favorecer la construcción de un significado robusto.

\section{Referencias bibliográficas}

Almira, J. M. S., \& Laborde, J. M. (2015). Modelo Didáctico para la Formación Axiológica a través de la Resolución de Problemas Matemáticos. Revista Digital: Matemática, Educación e Internet, 4(1).

Ausubel, D. P., Novak, J. D., \& Hanesian, H. (1976). Psicología educativa: un punto de vista cognoscitivo (Vol. 3). México: Trillas.

Ausubel, D. (1983). Teoría del aprendizaje significativo. Fascículos de CEIF, 1. 5.

Auzmendi Escribano, E. (1992). Las actitudes hacia la matemática-estadística en las enseñanzas media y universitaria. Características y medición. Ed mensajero. España.

Bransford, J. D., Sherwood, R. D., \& Sturdevant, T. (1987). Teaching thinking and problem solving. WH Freeman/Times Books/Henry Holt \& Co. 
Casal, J., \& Mateu, E. (2003). Tipos de muestreo. Rev. Epidem. Med. Prev, 1(1), 3-7. P.5.

De Losada M. F. (2012). Corrientes del pensamiento matemático del siglo XX. Primera parte: fundamentación. U. Antonio Nariño. p. 85.

Flórez Ochoa, R. (1994). Modelos pedagógicos y enseñanza de las ciencias. Hacia una pedagogía del conocimiento. Bogotá, McGraw Hill.

Freudenthal, H. (1986). Didactical phenomenology of mathematical structures (Vol. 1). Springer Science \& Business Media.

Freudenthal, H. (1991). Revisiting Mathematics Education: China Lectures, Kluwer, Dordrecht, Reidel Publishing Co.

Godino, J. D. (2002). Un enfoque ontológico y semiótico de la cognición matemática. Recherches en didactique des Mathématiques, 22(2/3), 237-284.

Hersh, R. (1997). What is mathematics, really? London, Oxford University Press.

Hilbert, D. (1928). Die grundlagen der mathematik. In Die Grundlagen der Mathematik (pp. 1-21). Vieweg+ Teubner Verlag, Wiesbaden.

Huang, W. H. Y., \& Soman, D. (2013). Gamification of education. Research Report Series: Behavioural Economics in Action, Rotman School of Management, University of Toronto. P. 17

Lakatos, I., Feigl, H., Hall, R. J., Koertge, N., \& Kuhn, T. S. (1982). Historia de la ciencia y sus reconstrucciones racionales. Madrid: Tecnos.

Lakatos, I. (1986). Pruebas y refutaciones. Madrid, Alianza.

McNeil, A. J., Frey, R., \& Embrechts, P. (2015). Quantitative risk management: concepts, techniques and toolsrevised edition. Princeton university press, p. 85.

Markopoulus, A. Fragkou, A. Davim, J. (2015). Gamification in engineering education and professional training. International Journal of Mechanical Engineering Education. 2015, Vol. 43(2) 118-131

Nieto, J.H. (2005). Olimpiadas matemáticas: El arte de resolver problemas. Ed. CEC, S.A. Los libros de 51. Nieto, J.H. (2005). Olimpiadas matemáticas: El arte de resolver problemas. Ed. CEC, S.A. Los libros de El Nacional. ISBN: 980.388.239-2 El Nacional. ISBN: 980.388.239-2

Novales, A. (2017). Midiendo el riesgo en mercados financieros. Universidad Complutense de Madrid. 93.

Planas, N. (2006). Modelo de análisis de videos para el estudio de procesos de construcción de conocimiento matemático. Educación matemática, 18(1), 37-72.

Vigotsky, L. S. (1988). Interacción entre enseñanza y desarrollo. Selección de Lecturas de Psicología de las Edades I, 3.

Wilcoxon, F., Katti, S. K., \& Wilcox, R. A. (1970). Critical values and probability levels for the Wilcoxon rank sum test and the Wilcoxon signed rank test. Selected tables in mathematical statistics.

Pavlov, I. P. (1927). Conditioned reflexes. (G. V. Anrep, Trans.). London: Oxford University Press 
Pérez, D. (2015). Construcción de significado robusto para el concepto de área y caracterización del pensamiento geométrico involucrado en los estudiantes de sexto grado. (Tesis Doctoral). Universidad Antonio Nariño, Bogotá.

Piaget, J. (1976). Piaget's theory. In Piaget and his school (pp. 11-23). Springer, Berlin, Heidelberg.

Popper, K. (1986). La Lógica de la Investigación Científica. Madrid. Editorial Tecnos

Russell, B., Domingo, J. N., \& Wood, A. (1982). La evolución de mi pensamiento filosófico. Madrid. Alianza.

Suh, A., Wagner, C., \& Liu, L. (2018). Enhancing user engagement through gamification. Journal of Computer Information Systems, 58(3), 204-213.

Yunita, D. R., Maharani, A., \& Sulaiman, H. (2019, April). Identifying of Rigorous Mathematical Thinking on Olympic Students in Solving Non-routine Problems on Geometry Topics. In 3rd Asian Education Symposium (AES 2018). Atlantis Press.

Esta obra está bajo una Licencia Creative Commons Attribución-NoCommercial 4.0 International

\section{(cc) BY-NC}

